\title{
Localização de fontes sonoras: a importância das diferenças dos limiares auditivos interaurais
}

\author{
Sound localization: the relevance of interaural differences \\ in auditory thresholds
}

\author{
Aline Cabral de Oliveira ${ }^{1}$, Luciana Pinto Rocha ${ }^{2}$, Vivian Passos Lima ${ }^{3}$, Leônidas Morais Netto ${ }^{4}$, \\ Pedro de Lemos Menezes ${ }^{5}$, José Fernando Colafêmina ${ }^{6}$
}

\begin{abstract}
RESUMO
Objetivo: Esclarecer a relação das diferenças entre os limiares auditivos das orelhas direita e esquerda com o percentual de erros e acertos na localização de fontes sonoras em ambientes reverberantes. Métodos: Foram examinados 56 voluntários, sendo 33 homens e 23 mulheres, média de idade de 26,6 anos, submetidos aos seguintes testes: otoscopia, audiometria tonal, imitanciometria e emissões otoacústicas. Todos os voluntários apresentaram audição dentro dos padrões de normalidade. Posteriormente, os indivíduos foram submetidos ao teste de localização sonora espacial, onde foram utilizadas ondas quadradas com frequiências fundamentais centradas em $500 \mathrm{~Hz}, 2000 \mathrm{~Hz}$ e $4000 \mathrm{~Hz}$. Os estímulos foram apresentados quinze vezes em cada uma das treze posições espaciais do aparelho utilizado, em cada uma das freqüências escolhidas. Assim, foram apresentados 585 estímulos, no total, por pessoa. Resultados: Na amostra estudada, encontrou-se como resultado, idade entre 18 e 50 anos, média da diferença dos limiares auditivos entre as orelhas de 6,81 dB e percentual de acertos na localização sonora dentro dos padrões normais. A correlação entre as diferenças interaurais e os acertos na localização, no entanto, não foi significativa. Conclusões: O presente estudo demonstrou que, em pessoas com audição normal, as variações dos limiares auditivos e as diferenças entre as orelhas até 10 dB não interferem na localização de fontes sonoras.
\end{abstract}

Descritores: Limiar auditivo; Audição; Orelha

\section{INTRODUÇÃO}

A percepção da direção dos sons é um fenômeno que ocorre de maneira inconsciente e está presente desde cedo no ciclo de vida humano, sendo uma função relacionada com a sobrevivência: por volta de seis meses de idade um bebê normal já

Trabalho realizado no Laboratório de Instrumentação e Acústica (LIA) da Universidade Estadual de Ciências da Saúde de Alagoas - UNCISAL - Maceió (AL), Brasil.

(1) Pós-graduanda em Otorrinolaringologia da Universidade de Medicina de Ribeirão Preto da Universidade de São Paulo - FMRP-USP - Ribeirão Preto (SP), Brasil.

(2) Acadêmica da Universidade Estadual de Ciências da Saúde de Alagoas UNCISAL - Maceió (AL), Brasil.

(3) Fonoaudióloga clínica, graduada pela Universidade Estadual de Ciências da Saúde de Alagoas - UNCISAL - Maceió (AL), Brasil.

(4) Fonoaudiólogo clínico, graduado pela Universidade Estadual de Ciências da Saúde de Alagoas - UNCISAL - Maceió (AL), Brasil.

(5) Mestre, Professor Assistente da Faculdade de Fonoaudiologia da Universidade Estadual de Ciências da Saúde de Alagoas - UNCISAL - Maceió (AL), Brasil.

(6) Livre-docente, Professor Assistente da Faculdade de Medicina de Ribeirão Preto da Universidade de São Paulo - USP - Ribeirão Preto (SP), Brasil.

Endereço para correspondência: Pedro de Lemos Menezes. R. Dr Antônio Cansanção, 55/703, Ponta Verde, Maceió - AL, CEP 57035-190. E-mail: son@hotlink.com.br

Recebido em: 13/6/2007; Aceito em: 8/2/2008 é capaz de localizar sons a sua volta ${ }^{(1)}$

Para que ocorra a percepção da direção do som, deve haver a discriminação de diferenças muito sutis de intensidade, frequiência e tempo. Geralmente, a localização sonora espacial depende da análise das diferenças, com relação a esses parâmetros, entre os sons que chegam às duas orelhas ${ }^{(2-6)}$.

A localização de fontes sonoras acontece, sobretudo, pela influência de características físicas da onda sonora, que geram diferenças no espectro dessa onda entre as orelhas, as chamadas diferenças interaurais ${ }^{(7-8)}$. Assim, dois fatores principais aparecem, as diferenças de fase entre as orelhas, diferença de tempo interaural (DTI) e as diferenças de intensidade entre as orelhas, diferença de intensidade interaural (DII).

O padrão de comparação das pressões entre as orelhas direita e esquerda é a diferença de intensidade interaural (DII), com limiar de aproximadamente $0,5 \mathrm{~dB}^{(9-11)}$. A localização por DII é melhor quando a frequiência do som possui ondas com comprimentos inferiores ao diâmetro da cabeça - 17,5 cm (2000 Hz) - provocando uma diferença de pressão entre as orelhas, denominado efeito sombra. Entretanto, este só tem bom funcionamento para frequiências superiores a $4000 \mathrm{~Hz}$ (comprimento de onda aproximadamente duas vezes menor que o diâmetro da cabeça). Este tipo de localização é mais eficiente quando a sombra resulta em uma diferença interaural de mais de $20 \mathrm{~dB}^{(8)}$. 
A diferença de fase da onda sonora entre as orelhas é definida como sendo o tempo que transcorre para uma área de compressão de partículas, uma crista, após ter atingido uma orelha, atingir a outra ${ }^{(12-14)}$. Esta diferença de fase corresponde à diferença de tempo interaural (DTI), a qual depende do raio da cabeça, da velocidade do som e do ângulo horizontal entre a fonte sonora e a ponta do nariz do indivíduo. As diferenças de fase entre as orelhas estão presentes apenas quando a frequiência do estímulo sonoro a ser localizado é menor que 1500 $\mathrm{Hz}$, pois, desta maneira, o comprimento da onda sonora será maior do que o diâmetro da cabeça, favorecendo a chegada da onda fora de fase, principalmente, quando a fonte sonora encontra-se à extrema direita ou à extrema esquerda ${ }^{(9)}$.

Com relação às diferenças interaurais, alguns autores ${ }^{(2,15)}$ concluem que existe uma faixa de freqüências, entre $1500 \mathrm{e}$ $3000 \mathrm{~Hz}$, em que a localização sonora se torna difícil, pois os comprimentos de ondas aproximam-se do diâmetro da cabeça, possibilitando a chegada do som praticamente em fase e com a mesma intensidade nas duas orelhas.

A excitação auditiva varia logaritmicamente, então, a intensidade sonora deve ser medida através do logaritmo da pressão ou da intensidade de energia ${ }^{(16)}$. Assim, a potência de um som é proporcional à pressão multiplicada por si mesma, ou seja, ao quadrado da pressão. Por este motivo, se a pressão for duplicada, a potência será quatro vezes maior. Portanto, para expressar coeficientes de pressão em decibel, a fórmula poderá ser escrita da seguinte maneira:

$$
d B=10 \log \frac{(P)^{2}}{\left(P_{0}\right)^{2}}
$$

ou

$$
d B=20 \log \frac{P}{P_{0}}
$$

Para minimizar a interferência das assimetrias interaurais, estudos que envolvem a localização de fontes sonoras, de um modo geral, estabelecem um valor máximo de $10 \mathrm{~dB}$ entre os limiares auditivos das duas orelhas, a exemplo de trabalhos anteriores ${ }^{(17)}$. Entretanto, sabe-se que diferenças de $6 \mathrm{~dB}$ já representam uma sensibilidade duas vezes maior para uma orelha em relação à outra ${ }^{(16,18)} \mathrm{e} 10 \mathrm{~dB}$, aproximadamente dez vezes, o que, teoricamente, poderá dificultar a localização por DII.

Desta maneira, o presente estudo teve como objetivo identificar os prejuízos relacionados a assimetrias entre as duas orelhas de ouvintes normais, mesmo que as mesmas não ultrapassem $10 \mathrm{~dB}$. Então, os valores encontrados entre os limiares auditivos foram correlacionados com o percentual de acertos na localização de fontes sonoras.

O esclarecimento de aspectos como este é importante para a compreensão da fisiologia do sistema auditivo e também para o fortalecimento da Fonoaudiologia, e da Audiologia em especial, uma Ciência relativamente nova, por meio da sua inserção no desenvolvimento de pesquisas básicas e, conseqüentemente, na produção do conhecimento.

\section{MÉTODOS}

O protocolo desta pesquisa está baseado na legislação pertinente, Resolução № 196/96, do Conselho Nacional de Saúde, do Ministério da Saúde, para estudos com seres humanos e foi aprovado pelo Comitê de Ética em Pesquisa da Universidade Estadual de Ciências da Saúde de Alagoas - UNCISAL, com protocolo ${ }^{\circ} 118$. Os exames foram realizados no Laboratório de Audiologia Dr. Marco Antônio Mota Gomes e no Laboratório de Instrumentação e Acústica (LIA), ambos na UNCISAL.

Os critérios de inclusão dos voluntários foram: a) limiares auditivos iguais ou inferiores a $20 \mathrm{~dB}$ NA (ANSI -1969), nas frequiências de 250 a $8000 \mathrm{~Hz}$, com diferenças entre as orelhas por frequiência iguais ou inferiores a $10 \mathrm{~dB}$; b) timpanogramas do tipo A, com pico em 0 da $\mathrm{Pa}$; c) reflexos estapedianos presentes; d) idades entre 18 e 50 anos. Os critérios de exclusão foram: a) exposição a ruído ocupacional ou de lazer; b) cirurgias no ouvido; c) mais de três infecções de ouvido no ano corrente; d) uso de medicação ototóxica; e) casos hereditários de surdez; f) experiência anterior com testes de localização sonora espacial.

Inicialmente, foi realizado um teste piloto (quatro homens e quatro mulheres) para adequação dos procedimentos. Em seguida, foram examinados 56 voluntários ( 33 homens e 23 mulheres).

Depois do questionário de triagem, os participantes preencheram e assinaram um "termo de consentimento livre e esclarecido", após terem recebido explicações sobre o estudo. Antes dos testes com o Analisador de percepção Sonora Espacial (APSE) foram realizados os seguintes procedimentos:

Otoscopia para a inspeção do meato acústico externo e da integridade da membrana timpânica, com um otoscópio da marca Welch Allyn ${ }^{\circledR} 29090$, com espéculos esterilizados.

Audiometria tonal liminar, com audiômetro da marca Interacoustics ${ }^{\circledR}$, modelo AC40, com tom puro pulsátil, em cabina acústica. Os limiares foram pesquisados por meio da técnica descendente, com intervalos de $10 \mathrm{~dB}$ e a confirmação das respostas pela técnica ascendente, com intervalos de $5 \mathrm{~dB}$. Foram avaliadas as seguintes freqüências: $500 \mathrm{~Hz}, 1000 \mathrm{~Hz}$; $2000 \mathrm{~Hz}, 4000 \mathrm{~Hz}, 8000 \mathrm{~Hz}, 9000 \mathrm{~Hz}, 10000 \mathrm{~Hz}, 12500 \mathrm{~Hz}$, $14000 \mathrm{~Hz}$ e $16000 \mathrm{~Hz}$. A cabina acústica seguiu as recomendações da ANSI 3.1 - 1991.

Imitanciometria, com aparelho da marca $\mathrm{MAICO}^{\circledR}$ modelo MI34, seguida da timpanometria e da pesquisa dos reflexos estapédicos.

O teste de localização sonora foi realizado em uma sala reverberante $(3 \times 3 \times 3)$ do LIA - UNCISAL, com níveis ambientais de ruído de aproximadamente $41 \mathrm{~dB}(\mathrm{~A})$ e completamente iluminada. O equipamento utilizado na testagem da localização sonora espacial dos participantes foi o mesmo das pesquisas de Menezes et al. ${ }^{(3)}$.

Foram utilizadas ondas quadradas com freqüências fundamentais, $500 \mathrm{~Hz}, 2000 \mathrm{~Hz}$, e $4000 \mathrm{~Hz}$. Para o grupo piloto foram apresentados 20 estímulos em cada uma das 13 posições, nas três freqüências, com um total de 780 estímulos por pessoa analisada.

No grupo teste, foram apresentados 15 estímulos em cada uma das 13 posições, nas três frequiências utilizadas, totalizando 585 estímulos para cada voluntário. A intensidade foi fixada em $70 \mathrm{~dB}(\mathrm{~A})$, medida no centro da estrutura metálica do aparelho 
por um analisador de nível de pressão sonora da marca Mini$\mathrm{pa}^{\circledR}$, modelo MSL - 1351C. O som produzido foi registrado pelo software Sound Forge para que os harmônicos pudessem ser analisados. Os estímulos aplicados tiveram duração de um segundo e o tempo máximo de resposta esperado foi de quatro segundos, além do qual tal resposta era considerada nula. Os tempos foram marcados com um cronômetro Casio ${ }^{\circledR}$.

Foi utilizada uma freqüência por vez, escolhida antecipadamente de forma aleatória. A ordem de estimulação dos alto-falantes - 195 estímulos por freqüência - foi feita com a ajuda do Software Random Number Generator (1997), sendo diferente para cada participante. Para o registro das respostas foram utilizados formulários impressos com a ordem dos estímulos, impressa pelo programa citado acima. No teste piloto foram utilizados os mesmos parâmetros.

As pessoas testadas deveriam indicar em um controle ou console auxiliar, que possuía botões com a representação espacial dos alto-falantes, a origem da fonte sonora de acordo com a seqüência apresentada pelo pesquisador. Esta seqüência era gerada por um controle de comando, que também possuía 13 botões de interruptores push-bottom, representando espacialmente estes alto-falantes. Acima de cada interruptor, neste controle de comando, existia um led de luz vermelha, que acendia de acordo com as respostas dos participantes. Todos os testes de localização sonora foram realizados pelo pesquisador responsável, que contava com o auxílio de um aluno de iniciação científica.

Após os dados obtidos serem caracterizados com a utilização de técnicas de estatística descritiva, foi aplicado o teste de Shapiro-Wilk para observar a normalidade da amostra. Em seguida, o teste de correlação bivariada, no qual foi avaliado o grau de relacionamento linear por meio do coeficiente de Pearson, para correlacionar os acertos na localização e as diferenças entre as orelhas. Os cálculos foram obtidos por meio do software SPSS, na versão 15.0.

\section{RESULTADOS}

A amostra estudada, formada por 56 voluntários, sendo 33 homens e 23 mulheres, teve média de idade de 26,6 anos e desvio padrão de 9,04 anos. Na Figura 1 podem ser observadas as médias de acertos na localização sonora, por freqüência

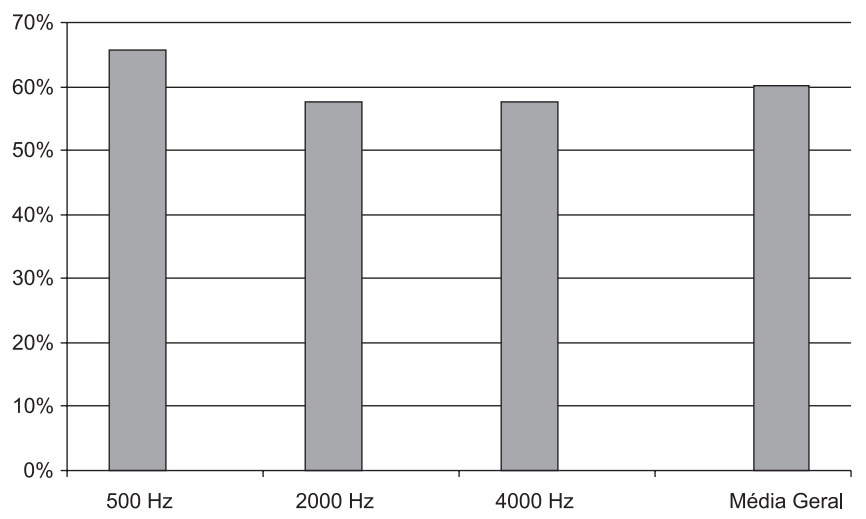

Figura 1. Percentual médio de acertos na localização sonora por freqüência de estimulação de estimulação e a média geral de acertos. Observa-se um maior percentual de acertos com estímulos na frequiência de $500 \mathrm{~Hz}$.

A Figura 2 mostra a média das diferenças em módulo dos limiares auditivos entre as orelhas: direita e esquerda. Observase que estas diferenças estão entre 4,3 e $9 \mathrm{~dB}$, com média de $6,81 \mathrm{~dB}$. O teste estatístico de correlação bivariada entre os acertos na localização de fontes sonoras, por frequiência (500, 2000 e $4000 \mathrm{~Hz}$ ) e a média geral, com a média das diferenças em módulo entre as orelhas, também por freqüência, mostrou valores de $\mathrm{r}$ (coeficiente de correlação) menores que 0,35 e valores de $\mathrm{p}$ (probabilidade) maiores que 0,05 , como pode ser visto na Tabela 1. Desse modo, constata-se que não existiu correlação significativa entre as variáveis estudadas.

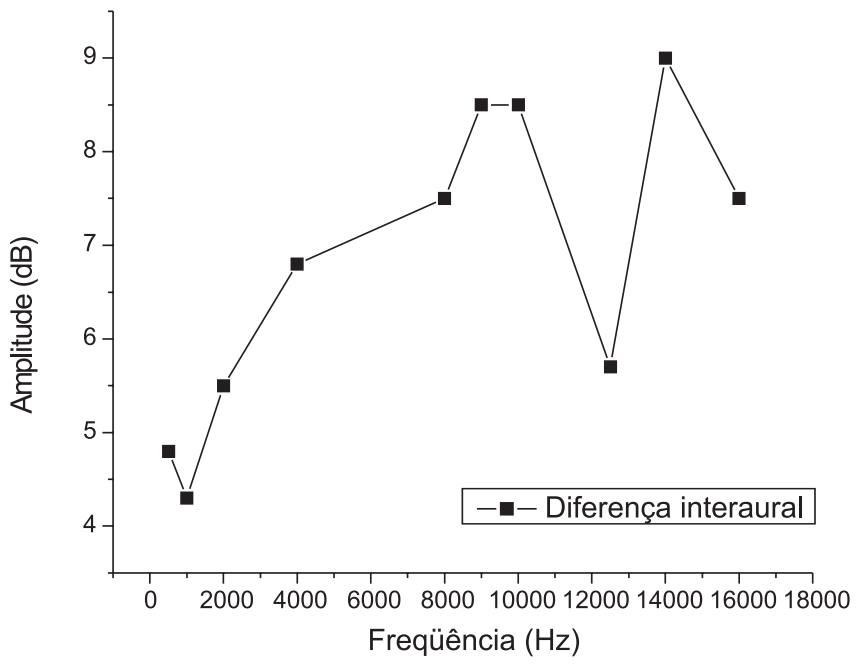

Figura 2. Média das diferenças em módulo entre as orelhas

Tabela 1. Correlação bivariada entre os acertos na localização de fontes sonoras, por freqüência $(500,2000$ e $4000 \mathrm{~Hz})$ e a média geral, com a média das diferenças em módulo entre as orelhas

\begin{tabular}{|c|c|c|c|c|c|}
\hline \multirow{2}{*}{$\begin{array}{l}\text { Média das } \\
\text { Diferenças }\end{array}$} & \multicolumn{5}{|c|}{ Localização sonora } \\
\hline & & $500 \mathrm{~Hz}$ & $2000 \mathrm{~Hz}$ & $4000 \mathrm{~Hz}$ & Média \\
\hline \multirow[t]{2}{*}{$500 \mathrm{~Hz}$} & $r$ & $-0,01$ & $-0,01$ & $-0,05$ & $-0,02$ \\
\hline & $p$ & 0,89 & 0,97 & 0,72 & 0,85 \\
\hline \multirow[t]{2}{*}{$1000 \mathrm{~Hz}$} & $r$ & 0,15 & 0,19 & 0,21 & 0,19 \\
\hline & $p$ & 0,28 & 0,16 & 0,12 & 0,17 \\
\hline \multirow[t]{2}{*}{$2000 \mathrm{~Hz}$} & $r$ & $-0,02$ & 0,01 & $-0,01$ & $-0,01$ \\
\hline & $p$ & 0,86 & 0,96 & 0,96 & 0,95 \\
\hline \multirow[t]{2}{*}{$4000 \mathrm{~Hz}$} & r & $-0,11$ & $-0,17$ & $-0,15$ & $-0,15$ \\
\hline & $p$ & 0,43 & 0,23 & 0,26 & 0,29 \\
\hline \multirow[t]{2}{*}{$8000 \mathrm{~Hz}$} & $r$ & 0,17 & 0,16 & 0,18 & 0,18 \\
\hline & $p$ & 0,21 & 0,24 & 0,19 & 0,20 \\
\hline \multirow[t]{2}{*}{$9000 \mathrm{~Hz}$} & $r$ & 0,02 & 0,05 & $-0,02$ & 0,02 \\
\hline & $\mathrm{p}$ & 0,88 & 0,68 & 0,89 & 0,89 \\
\hline \multirow[t]{2}{*}{$10000 \mathrm{~Hz}$} & r & $-0,15$ & $-0,11$ & $-0,09$ & $-0,12$ \\
\hline & $\mathrm{p}$ & 0,28 & 0,42 & 0,49 & 0,37 \\
\hline \multirow[t]{2}{*}{$12500 \mathrm{~Hz}$} & $r$ & 0,25 & 0,22 & 0,18 & 0,23 \\
\hline & $\mathrm{p}$ & 0,06 & 0,10 & 0,19 & 0,09 \\
\hline \multirow[t]{2}{*}{$14000 \mathrm{~Hz}$} & $r$ & $-0,11$ & $-0,07$ & $-0,09$ & $-0,09$ \\
\hline & $p$ & 0,41 & 0,63 & 0,53 & 0,49 \\
\hline \multirow[t]{2}{*}{$16000 \mathrm{~Hz}$} & $r$ & 0,26 & 0,26 & 0,32 & 0,28 \\
\hline & $p$ & 0,06 & 0,06 & 0,07 & 0,06 \\
\hline
\end{tabular}

Legenda: $r=$ coeficiente de correlação; $p=$ valores de $p$ (probabilidade) 


\section{DISCUSSÃO}

Os resultados encontrados para a localização de fontes sonoras, com maior número de acertos na freqüência de $500 \mathrm{~Hz}$, já eram esperados, uma vez que nesta freqüência, as diferenças de tempos interaurais são mais eficientes. Nas outras duas frequiências, a localização é mais difícil pela diminuição de todos os tipos de diferenças interaurais, fato bastante descrito na literatura ${ }^{(2,8-9,15)}$.

As diferenças de intensidades entre as orelhas, neste estudo, variam de 4,3 a $9 \mathrm{~dB}$, o que representa uma sensibilidade até praticamente três vezes maior para uma orelha em relação à outra $^{(16,18)}$. Apesar dos limiares tonais poderem ser percebidos com diferenças mínimas de $0,5 \mathrm{~dB}^{(9)}$, diversos autores em seus estudos utilizaram diferenças entre limiares das orelhas de até $10 \mathrm{~dB}^{(3-4)}$. Entretanto, esse valor representa uma diferença entre as orelhas de aproximadamente 10 vezes, o que teoricamente poderia interferir na localização de fontes sonoras, sem considerar, contudo, possíveis adaptações do sistema auditivo.

A não existência da correlação entre os acertos na locali- zação sonora e as diferenças dos limiares auditivos entre as orelhas justifica a decisão de diversos autores em não excluir deste tipo de pesquisa, voluntários com diferença interaural de até $10 \mathrm{~dB}$.

A efetividade na localização de fontes sonoras por diferença de intensidade interaural acontece quando a sombra entre as orelhas tem mais de $20 \mathrm{~dB}^{(8)}$, justificando, mais uma vez, os resultados encontrados neste trabalho.

Um dado que será estudado futuramente é que a diferença de aproximadamente $6 \mathrm{~dB}$ não foi constante para todas as frequiências, isso pode ter sido a explicação por não ter havido piora nos acertos na localização do som. Esse fato já está sendo investigado e será apresentado em estudo posterior.

\section{CONCLUSÃO}

As diferenças entre os limiares da orelha direita e da orelha esquerda entre 4,3 e $9 \mathrm{~dB}$ não interferem no percentual de acertos na localização de fontes sonoras.

\begin{abstract}
Purpose: To relate the differences between left and right ears' auditory thresholds and rate of correct/incorrect responses in sound localization tasks placed in reverberant environments. Methods: The following tests were carried out: otoscopy, pure tone audiometry, impedanciometry and otoacoustic emissions. All 56 volunteers presented normal hearing results in all tests. After that, the volunteers were submitted to a sound localization test, in which square waves of $500 \mathrm{~Hz}, 2000 \mathrm{~Hz}$ and $4000 \mathrm{~Hz}$ were used. Stimuli were presented fifteen times in each one of the thirteen space positions of the device used, for each frequency tested. Therefore, a total number of 585 stimuli were presented for each subject. Results: Data analysis showed that the sample had subjects with ages between 18 and 50 years, the average difference between right and left ears' auditory thresholds was $6,81 \mathrm{~dB}$, and percentage of correct answers on the sound localization test was within normal values. No significant correlations were found between interaural differences and localization abilities. Conclusions: The present study showed that, for people with normal hearing, the auditory thresholds variations and interaural differences until $10 \mathrm{~dB}$ do not interfere on sound localization.
\end{abstract}

Keywords: Auditory threshold; Hearing; Ear

\title{
REFERÊNCIAS
}

1. Furst M, Bresloff I, Levine RA, Merlob PL, Attias JJ. Interaural time coincidence detectors are present at birth: evidence from binaural interaction. Hear Res. 2004;187(1-2):63-72.

2. Blauert J. Spatial hearing : the psychophysics of human sound localization. London: MIT Press; 1999. p. 30-245.

3. Menezes PL, Soares IA, Caldas Neto S, Maciel R, Motta MA. Estudo da localização sonora em ouvintes normais. J Bras Fonoaudiol. 2003;4(15):109-13.

4. Menezes PL, Soares IA, Caldas Neto S, Maciel R, Motta MA. Localização sonora: Uma nova perspectiva de estudo para a audiologia. Fonoaudiol Brasil. 2003;2(3):28-35.

5. Carlile S, Martin R, McAnally K. Spectral information in sound localization. Int Rev Neurobiol. 2005;70:399-434.

6. Nix J, Hohmann V. Sound source localization in real sound fields based on empirical statistics of interaural parameters. J Acoust Soc Am. 2006;119(1):463-79.

7. Faller C, Merimaa J. Source localization in complex listening situations: selection of binaural cues based on interaural coherence. J Acoust Soc Am. 2004;116(5):3075-89.
8. Simon HJ. Bilateral amplification and sound localization: then and now. J Rehabil Res Dev. 2005;42(4 Suppl 2):117-32.

9. Langendijk EH, Kistler DJ, Wightman FL. Sound localization in the presence of one or two distracters. J Acoust Soc Am. 2001;109(5 Pt 1):2123-34.

10. Després O, Candas V, Dufour A. Auditory compensation in myopic humans: involvement of binaural, monaural, or echo cues? Brain Res. 2005;1041(1):56-65.

11. Abel SM, Tikuisis C. Sound localization with monocular vision. Defence Res Develop. 2006;66(8):932-44.

12. Macpherson EA, Middlebrooks JC. Listener weighting of cues for lateral angle: the duplex theory of sound localization revisited. J Acoust Soc Am. 2002;111(5 Pt 1):2219-36.

13. Aarabi P, Mavandadi S. Robust sound localization using conditional time-frequency histograms. Inform Fusion. 2003;4(2):111-2.

14. Shinn-Cunningham BG, Kopco N, Martin TJ. Localizing nearby sound sources in a classroom: binaural room impulse responses. J Acoust Soc Am. 2005;117(5):3100-15. 
15. Sabin AT, Macpherson EA, Middlebrooks JC. Human sound localization at near-threshold levels. Hear Res. 2005;199(1-2):124-34.

16. Bistafa SR. Acústica aplicada ao controle do ruído. São Paulo: Blucher; 2006. p. 73-185.

17. Oldfield SR, Parker SP. Acuity of sound localisation: a topography of auditory space. I. Normal hearing conditions. Perception. 1984;13(5):581-600.
18. García Callejo FJ, García Callejo F, Pena Santamaría J, Alonso Castañeira I, Sebastián Gil E, Marco Algarra J. [Hearing level and intensive use of mobile phones]. Acta Otorrinolaringol Esp. 2005;56(5):187-91. Spanish. 